\title{
Conditional linearizability criteria for a system of third-order ordinary differential equations
}

\author{
F. M. Mahomed ${ }^{a}$, I. Naeem ${ }^{a}$ and Asghar Qadir ${ }^{b}$ \\ ${ }^{a}$ Centre for Differential Equations, Continuum Mechanics and Applications \\ School of Computational and Applied Mathematics \\ University of the Witwatersrand \\ Wits 2050, South Africa \\ E-mail: Fazal.Mahomed@wits.ac.za \\ ${ }^{b}$ Centre for Advanced Mathematics and Physics \\ National University of Sciences and Technology \\ Campus of the College of Electrical and Mechanical Engineering \\ Peshawar Road, Rawalpindi, Pakistan and \\ Department of Mathematical Sciences \\ King Fahd University of Petroleum and Minerals \\ Dhahran 31261, Saudi Arabia \\ E-mail: aqadirs@comsats.net.pk
}

\begin{abstract}
We provide linearizability criteria for a class of systems of third-order ordinary differential equations (ODEs) that is cubically semi-linear in the first derivative, by differentiating a system of second-order quadratically semi-linear ODEs and using the original system to replace the second derivative. The procedure developed splits into two cases, those where the coefficients are constant and those where they are variables. Both cases are discussed and examples given.
\end{abstract}

\section{Introduction}

Linearization plays an important role in the study of nonlinear differential equations as there is no standard method to solve nonlinear ODEs. In 1883, Lie [1] gave the linearizability criteria for scalar second-order ordinary differential equations and he constructed the most general form of second-order ODEs reducible to linear ones by changing the dependent and independent variables. 
Lie also developed point transformation groups [2]. To the best of our knowledge Lie did not provide linearizability criteria either for second-order systems or for third-order scalar ODEs, leave alone systems of third order ODEs. Nor does this aspect appear in the literature. One can use the approximation (perturbation method) to convert nonlinear ODEs into linear ones [3] but one faces problems in the convergence of the series. A similar problem arises in numerical schemes. Contact transformations were used by Chern [4, 5] to reduce scalar third-order equations into the linear forms $u^{\prime \prime \prime}=0$ and $u^{\prime \prime}+u=0$. Grebot [6] used fibre preserving point transformations for mapping third-order ODEs to linear form. A generalization of this work was later performed by Neut and Petitot [7. Ibragimov and Meleshko 8] and Maleshko [9] also studied the reduction of scalar third-order ODEs to linear forms. Whereas Neut and Petitot and Ibragimov and Meleshko used the classical Lie method, Meleshko dealt with a sub-class of those equations in which the independent variable did not appear explicitly and hence one could convert to treating the function to be determined as the independent variable and and its derivative as the dependent variable, thereby reducing the order by one.

The linearizability criteria for a system of second order quadratically semi-linear ODEs were discussed by two of us [10], using the connection between the symmetries and the isometries of the system of the geodesic equations [11]. The criteria require that the curvature tensor be zero, i.e. the space be flat. Subsequently [13] a projection procedure of Aminova and Aminov [12] was followed, using the translational invariance of the geodetic parameter in the geodesic equation. A system of two dimensions was used to get a single cubically semi-linear ODE and Lie's results on linearization of scalar second order ODEs was also re-derived. Moreover, invariant criteria for a system of two cubically semi-linear second-order ODEs to be reducible to a linear system were obtained.

A method was developed [14] The purpose of this paper is to construct the linearizability conditions for a system of third-order ODEs, cubically semi-linear in the first derivative by differentiating the system of second order quadratically semi-linear ODEs and then setting the original system to replace the second derivative. The procedure employed here uses the derivative of the vector of the coefficients in the equation and solves a system of non-homogeneous algebraic equations for it. If this vector becomes zero, the procedure of solving the system by inverting a matrix cannot be used directly. As such, one has to go back to the system and solve it directly. This allows us to construct the linearizability conditions in all cases. Since there is no work on explicit linearizability criteria of systems of third order non-linear ODEs, we cannot compare our results with other works. However, there is a result for the number of classes of linearizable systems of ODEs, which is based on there being $3 n$ arbitrary constants for the system, using the classical Lie method. The class of linearizable equations obtained here is not contained in those classes as it is non-classical and does not have the required number of arbitrary constants. 
The outline of the work is as follows. In the next section we briefly mention the geometrical notation used and review the linearizability criteria for second order quadratically semi-linear systems. In section 3 we discuss the linearizability criteria for the general case for a system of third-order ODEs when at least one of the coefficients is variable. The special case when all the coefficients are constant is discussed in the next section. In section 5 we illustrate via some examples to verify our criteria. Concluding remarks are given in section 6 .

\section{Preliminaries}

We shall use the Einstein summation convention that repeated indices are summed over. The position vector, $x^{j},(j=1, \ldots, n)$ is assumed to be a function of a geodetic parameter $s$ and ' represents the derivative relative to $s$. The metric tensor will be denoted by $g_{i j}\left(x^{m}\right)$ and the partial derivative relative to $x^{j}$ by ",j". The Christoffel symbols $\Gamma_{j k}^{i}$ are defined in terms of the metric tensor by

$$
\Gamma_{j k}^{i}=\frac{1}{2} g^{i l}\left(g_{j l, k}+g_{k l, j}-g_{j k, l}\right) .
$$

The geodesic is given by the equation

$$
x^{a^{\prime \prime}}+\Gamma_{b c}^{a} x^{b^{\prime}} x^{c^{\prime}}=0
$$

The Riemann tensor is defined by

$$
R_{j k l}^{i}=\Gamma_{j l, k}^{i}-\Gamma_{j k, l}^{i}+\Gamma_{m k}^{i} \Gamma_{j l}^{m}-\Gamma_{m l}^{i} \Gamma_{j k}^{m}
$$

which satisfies

$$
\begin{gathered}
R_{j k l}^{i}=-R_{j l k}^{i}, \\
R_{j k l}^{i}+R_{k l j}^{i}+R_{l j k}^{i}=0,
\end{gathered}
$$

and

$$
R_{j k l ; m}^{i}+R_{j l m ; k}^{i}+R_{j m k ; l}^{i}=0 .
$$

The Riemann tensor in complete covariant form is

$$
R_{i j k l}=g_{i m} R_{j l k}^{m},
$$

and has the additional property

$$
R_{i j k l}=-R_{j i k l} .
$$


A general system of quadratically semi-linear second order ODEs can be written as

$$
x^{a^{\prime \prime}}+\gamma_{b c}^{a} x^{b^{\prime}} x^{c^{\prime}}+\beta_{b}^{a} x^{b^{\prime}}+\alpha^{a}=0,
$$

where $\gamma_{b c}^{a}, \beta_{b}^{a}, \alpha^{a}$ are functions of the $n$ independent variables. If $\beta_{b}^{a}, \alpha^{a}=0$ we will call it of geodesic type if the coefficients $\gamma_{b c}^{a}$ can be written as $\Gamma_{b c}^{a}$ for some metric tensor $g_{a b}$.

For example, a two-dimensional system of geodesic type is of the form

$$
\left\{\begin{array}{l}
x^{\prime \prime}=a(x, y) x^{\prime 2}+2 b(x, y) x^{\prime} y^{\prime}+c(x, y) y^{\prime 2}, \\
y^{\prime \prime}=d(x, y) x^{\prime 2}+2 e(x, y) x^{\prime} y^{\prime}+f(x, y) y^{\prime 2}
\end{array}\right.
$$

where we have written $x^{1}=x, x^{2}=y$ and

$$
\begin{aligned}
& \Gamma_{11}^{1}=-a, \quad \Gamma_{12}^{1}=-b, \quad \Gamma_{22}^{1}=-c, \\
& \Gamma_{11}^{2}=-d, \quad \Gamma_{12}^{2}=-e, \quad \Gamma_{22}^{2}=-f .
\end{aligned}
$$

The linearizability condition of [10] is that the curvature tensor constructed from these coefficients, regarded as Christoffel symbols, be zero, i. e. $R_{j k l}^{i}=0$. In terms of the coefficients of (3.2) we have

$$
\begin{gathered}
a_{y}-b_{x}+b e-c d=0, \\
b_{y}-c_{x}+\left(a c-b^{2}\right)+(b f-c e)=0, \\
d_{y}-e_{x}-(a e-b d)-\left(d f-e^{2}\right)=0, \\
(b+f)_{x}=(a+e)_{y} .
\end{gathered}
$$

\section{Linearization for third-order ODEs}

To obtain the criteria for the third order semi-linear system of ODEs we differentiate (2.2) to get

$$
x^{a^{\prime \prime \prime}}+2 \Gamma_{b c}^{a} x^{b^{\prime \prime}} x^{c^{\prime}}+\Gamma_{b c, d}^{a} x^{b^{\prime}} x^{c^{\prime}} x^{d^{\prime}}=0 .
$$


This is a total derivative system and may seem to be an artificial way of getting the third order systems. However, if we now replace the $x^{b^{\prime \prime}}$ in (3.1) using (2.2) we get the system

$$
x^{a^{\prime \prime \prime}}+\left[\Gamma_{(b c, d)}^{a}-2 \Gamma_{p(b}^{a} \Gamma_{c d)}^{p}\right] x^{b^{\prime}} x^{c^{\prime}} x^{d^{\prime}}=0
$$

which is not a total derivative system. Thus the general form will be a third order system of ODEs that is cubically semi-linear in the first derivative

$$
x^{a^{\prime \prime \prime}}+A_{b c d}^{a} x^{b^{\prime}} x^{c^{\prime}} x^{d^{\prime}}=0
$$

which will be linearizable if we can make the identification

$$
A_{b c d}^{a}=\Gamma_{(b c, d)}^{a}-2 \Gamma_{p(b}^{a} \Gamma_{c d)}^{p}
$$

In the case of a system of two third-order ODEs the linearizability conditions (2.13) - (2.16) are satisfied. Using (3.3) and (3.4) with the help of (2.13) - (2.16), we obtain

$$
\begin{gathered}
A_{111}^{1}=-a_{x}-2 a^{2}-2 b d, \\
A_{112}^{1}=-a_{y}-2 b_{x}-2(3 a b+2 b e+c d), \\
A_{122}^{1}=-\left(2 b_{y}+c_{x}\right)-2\left(a c+2 b^{2}+b f+2 c e\right), \\
A_{222}^{1}=-c_{y}-2(b c+c f), \\
A_{111}^{2}=-d_{x}-2 a d-2 d e, \\
A_{112}^{2}=-\left(d_{y}+2 e_{x}\right)-2\left(2 b d+a e+2 e^{2}+d f\right), \\
A_{122}^{2}=-\left(2 e_{y}+f_{x}\right)-2(c d+2 b e+3 e f), \\
A_{222}^{2}=-f_{y}-2 c e-2 f^{2} .
\end{gathered}
$$


Invoking $(2.13)$ - (2.16) we can write $(3.5)$ - (3.12) as

$$
\begin{aligned}
& a_{x}=-\left(A_{111}^{1}+2 a^{2}+2 b d\right), a_{y}=-\left(A_{122}^{1} / 3+2 a b+2 b e\right), \\
& b_{x}=-\left(A_{112}^{1} / 3+c d+2 a b+b e\right), b_{y}=-\left(A_{122}^{1} / 3+b^{2}+a c+c e+b f\right), \\
& c_{x}=-\left(A_{122}^{1} / 3+2 b^{2}+2 c e\right), c_{y}=-\left(A_{222}^{1} / 3+2 b c+2 c f\right), \\
& d_{x}=-\left(A_{111}^{2}+2 a d+2 d e\right), d_{y}=-\left(A_{112}^{2} / 3+2 b d+2 e^{2}\right), \\
& e_{x}=-\left(A_{112}^{2} / 3+d f+b d+a e+e^{2}\right), e_{y}=-\left(A_{122}^{2} / 3+c d+b e+2 e f\right), \\
& f_{x}=-\left(A_{122}^{2} / 3+2 b e+2 e f\right), f_{y}=-\left(A_{222}^{2}+2 c e+2 f^{2}\right) .
\end{aligned}
$$

Using the compatibility conditions and for convenience writing

$$
\begin{aligned}
& A_{111}^{1}=P, \quad A_{112}^{1}=Q, \quad A_{122}^{1}=R, \quad A_{222}^{1}=S, \\
& A_{111}^{2}=T, \quad A_{112}^{2}=U, \quad A_{122}^{2}=V, \quad A_{222}^{2}=W,
\end{aligned}
$$

the system (3.13) reduces to

$$
\begin{array}{r}
3 P_{y}-2 a Q-2 d R=Q_{x}-6 b P-2 e Q, \\
Q_{y}-a R-3 d S=R_{x}-3 c P-f Q, \\
R_{y}-2 b R-6 e S=3 S_{x}-2 c Q-2 f R, \\
3 T_{y}-2 a U-2 d V=U_{x}-6 b T-2 e U, \\
U_{y}-a V-3 d W=V_{x}-3 c T-f U, \\
V_{y}-2 b V-6 e W=3 W_{x}-2 c U-2 f V .
\end{array}
$$

For $n=2$ the system (3.4) is linearizable if it satisfies conditions (3.13) and (3.15), where $a, b, c, d, e$ and $f$ are given in the Appendix. Our procedure puts the derivatives of the $A_{j k l}^{i}$ as a vector on the right side of a system of linear equations and solves for the constraints on the coefficients by inverting the matrix of coefficients on the left side. If the vector on the right is zero, we cannot use this procedure to obtain the conditions on the coefficients. As such, for this procedure to work at least one of the coefficients in the equations must be variable. If all the coefficients in system (3.4) are constants then the derivatives of $A_{j k l}^{i}$ are zero. For this case we need to develop a separate procedure that is done in the next section.

\section{Special cases}

In the case $A_{j k l, m}^{i}=0$, the system (3.15) cannot be used for obtaining the coefficients by inverting the matrix. We have to solve the equations without resorting to that procedure. This system 
reduces to

$$
\begin{aligned}
& -a Q-d R+3 b P+e Q=0,-a R-3 d S+3 c P+f Q=0,-b R-3 e S+c Q+f R=0 \\
& -a U-d V+3 b T+e U=0,-a V-3 d W+3 c T+f U=0,-b V-3 e W+c U+f V=0
\end{aligned}
$$

and equations (3.5) - (3.12) become

$$
\begin{aligned}
A_{111}^{1}+2\left(a^{2}+b d\right)=0, A_{112}^{1}+6(a b+b e) & =0, \\
A_{112}^{1}+3(c d+2 a b+b e)=0, A_{122}^{1}+3\left(b^{2}+a c+c e+b f\right) & =0, \\
A_{122}^{1}+6\left(b^{2}+c e\right)=0, A_{222}^{1}+2(b c+c f) & =0, \\
A_{111}^{2}+2(a d+d e)=0, A_{112}^{2}+6\left(b d+e^{2}\right) & =0, \\
A_{112}^{2}+3\left(d f+b d+a e+e^{2}\right)=0, A_{122}^{2}+3(c d+b e+2 e f) & =0, \\
A_{122}^{2}+6(b e+e f)=0, A_{222}^{2}+2\left(c e+f^{2}\right) & =0 .
\end{aligned}
$$

Non-trivial solutions of (4.1) exist if

$$
\left|\begin{array}{cccccc}
-2 Q & 6 P & 0 & -2 R & 2 Q & 0 \\
-R & 0 & 3 P & -3 S & 0 & Q \\
0 & -2 R & 2 Q & 0 & -6 S & 2 R \\
-2 U & 6 T & 0 & -2 V & 2 U & 0 \\
-V & 0 & 3 T & -3 W & 0 & U \\
0 & -2 V & 2 U & 0 & -6 W & 2 V
\end{array}\right|=0 .
$$

This gives the linearizability criteria of (3.4) when all the coefficients are constants.

From system (4.1), we have

$$
\begin{array}{r}
c d-b e=0, b^{2}+c e-a c-b f=0, b d+e^{2}-d f-a e=0, \\
A_{111}^{1}=-2\left(a^{2}+b d\right), A_{112}^{1}=-6(a b+b e), \\
A_{122}^{1}=-6\left(b^{2}+c e\right), A_{222}^{1}=-2(b c+c f), \\
A_{111}^{2}=-2(a d+d e), A_{112}^{2}=-6\left(b d+e^{2}\right), \\
A_{122}^{2}=-6(b e+e f), A_{222}^{2}=-2\left(c e+f^{2}\right) .
\end{array}
$$

In order to solve system (4.2), the following cases are considered.

Case 1: $b=0$.

The following subcases arise. 
Case 1.1: $c=0, d=0$.

Case 1.1.1: $e=0$.

In this case we have

$$
\begin{gathered}
A_{112}^{1}=0, A_{122}^{1}=0, A_{222}^{1}=0, A_{111}^{2}=0, A_{112}^{2}=0, A_{122}^{2}=0, \\
A_{111}^{1}=-2 a^{2} A_{222}^{2}=-2 f^{2} .
\end{gathered}
$$

Hence all the $A_{j k l}^{i}$ are zero except $A_{111}^{1}$ and $A_{222}^{2}$, which must be non-positive since $a$ and $f$ are real.

Case 1.1.2: $e \neq 0$.

Simple manipulations yield

$$
\begin{gathered}
A_{112}^{1}=0, A_{122}^{1}=0, A_{222}^{1}=0, A_{111}^{2}=0, \\
A_{111}^{1}=-2 a^{2}, A_{112}^{2}=-6 a^{2}, A_{122}^{2}=-6 a f, A_{222}^{2}=-2 f^{2},
\end{gathered}
$$

which yield

$$
\begin{gathered}
a^{2}=-A_{111}^{1} / 2, f^{2}=-A_{222}^{2} / 2 \\
\left(A_{122}^{2}\right)^{2}-9 A_{111}^{1} A_{222}^{2}=0, A_{112}^{2}-3 A_{111}^{1}=0 .
\end{gathered}
$$

This further requires $A_{111}^{1}, A_{112}^{2}, A_{222}^{2} \leq 0$.

Case 1.2: $c=0, d \neq 0$.

Straightforward calculations lead to

$$
e^{2}-d f-a e=0
$$

and

$$
\begin{gathered}
A_{112}^{1}=0, A_{122}^{1}=0, A_{222}^{1}=0, \\
A_{111}^{1}=-2 a^{2}, A_{111}^{2}=-2(a d+d e), \\
A_{112}^{2}=-6 e^{2}, A_{122}^{2}=-6 e f, A_{222}^{2}=-2 f^{2},
\end{gathered}
$$


which yield

$$
\begin{gathered}
a^{2}=-\frac{A_{111}^{1}}{2}, d^{2}=\frac{3\left(A_{111}^{2}\right)^{2}}{2\left[-3 A_{111}^{1}-A_{112}^{2} \pm 2 \sqrt{3 A_{111}^{1} A_{112}^{2}}\right]}, \\
e^{2}=-A_{112}^{2} / 6, f^{2}=-A_{222}^{2} / 2,
\end{gathered}
$$

with conditions

$$
\begin{gathered}
\left(A_{122}^{2}\right)^{2}-3 A_{112}^{2} A_{222}^{2}=0 \\
3 A_{111}^{2}=\left[ \pm \sqrt{\frac{A_{111}^{1}}{A_{222}^{2}}}+\frac{A_{112}^{2}}{A_{122}^{2}}\right]\left[A_{112}^{2} \pm \sqrt{3 A_{111}^{1} A_{112}^{2}}\right]
\end{gathered}
$$

Thus we require that $A_{111}^{1}, A_{112}^{2} \leq 0, A_{222}^{2}<0$.

Case 1.3: $c \neq 0, d=0$.

We find that $e=a$ and

$$
\begin{gathered}
A_{112}^{1}=0, A_{111}^{2}=0, \\
A_{111}^{1}=-2 a^{2}, A_{122}^{1}=-6 a c, A_{222}^{1}=-2 c f, \\
A_{112}^{2}=-6 a^{2}, A_{122}^{2}=-6 a f, A_{222}^{2}=-2\left(a c+f^{2}\right),
\end{gathered}
$$

which yield

$$
\begin{gathered}
a^{2}=-\frac{A_{111}^{1}}{2}, c^{2}=-\frac{\left(A_{122}^{1}\right)^{2}}{18 A_{111}^{1}}, f^{2}=-\frac{\left(A_{122}^{2}\right)^{2}}{18 A_{111}^{1}}, \\
A_{112}^{2}-3 A_{111}^{1}=0,9 A_{111}^{1} A_{222}^{2}-3 A_{111}^{1} A_{122}^{1}-\left(A_{122}^{2}\right)^{2}=0, \\
81\left(A_{111}^{1}\right)^{2}\left(A_{222}^{1}\right)^{2}-\left(A_{122}^{1}\right)^{2}\left(A_{122}^{2}\right)^{2}=0 .
\end{gathered}
$$

In this case we need to have $A_{111}^{1}<0, A_{112}^{2} \leq 0$.

Case 2: $b \neq 0$.

The subcases are as follows.

Case 2.1: $d=0$.

In this case we find that

$$
e=0, b^{2}-a c-b f=0,
$$


and

$$
\begin{gathered}
A_{111}^{2}=0, A_{112}^{2}=0, A_{122}^{2}=0, \\
A_{111}^{1}=-2 a^{2}, A_{112}^{1}=-6 a b, A_{122}^{1}=-6 b^{2}, \\
A_{222}^{1}=-2(b c+c f), \quad A_{222}^{2}=-2 f^{2},
\end{gathered}
$$

which imply that

$$
\begin{array}{r}
a^{2}=-A_{111}^{1} / 2, b^{2}=-A_{122}^{1} / 6, f^{2}=-A_{222}^{2} / 2, \\
c^{2}=\frac{3\left(A_{222}^{1}\right)^{2}}{2\left[-3 A_{222}^{2}-A_{122}^{1} \pm 2 \sqrt{3 A_{122}^{1} A_{222}^{2}}\right]}
\end{array}
$$

with extra conditions on $A_{j k l}^{i}$ given by

$$
\begin{gathered}
\left(A_{112}^{1}\right)^{2}-3 A_{111}^{1} A_{122}^{1}=0 \\
3 A_{222}^{1}=\left[ \pm \sqrt{\frac{A_{222}^{2}}{A_{111}^{1}}}+\frac{A_{122}^{1}}{A_{112}^{1}}\right]\left[A_{122}^{1} \pm \sqrt{3 A_{122}^{1} A_{222}^{2}}\right] .
\end{gathered}
$$

and $A_{111}^{1}<0, A_{122}^{1}, A_{222}^{2} \leq 0$.

Case 2.2: $d \neq 0, e=c d / b$.

We find that

$$
b^{3}+c^{2} d-b^{2} f-a b c=0, b^{3} d+c^{2} d^{2}-b^{2} d f-a b c d=0,
$$

and

$$
\begin{gathered}
A_{111}^{1}=-2\left(a^{2}+b d\right), A_{112}^{1}=-6(a b+b e), A_{122}^{1}=-6\left(b^{2}+c e\right), \\
A_{222}^{1}=-2(b c+c f), A_{111}^{2}=-2(a d+d e), A_{112}^{2}=-6\left(b d+e^{2}\right), \\
A_{122}^{2}=-6(b e+e f), A_{222}^{2}=-2\left(c e+f^{2}\right) .
\end{gathered}
$$

In order to construct the linearizability conditions for above system one has to replace $e=\lambda \frac{d}{b}$ or $e=\lambda \frac{c}{b}$ to get a $5 \times 5$ matrix and the determinant of the resultant matrix must be zero. 


\section{$5 \quad$ Examples}

To test our linearization criteria, we utilize the following examples.

1. For the case when at least one of the coefficients is variable e.g. $P=0, Q=0, R=3, S=$ $0, T=0, U=-6 / x^{2}, V=0, W=2$, the system of two third-order equations is linearizable as it satisfies conditions (3.13)-(3.15) with $a, b, c, d, e$ and $f$ given in the Appendix.

The following set of six equations can be obtained from [10] by using the values of $a, b, c, d, e$ and $f$

$$
\begin{gathered}
p_{x}=0, q_{x}=q / x, r_{x}=2 r / x, \\
p_{y}=2 q / x, q_{y}=-x p+r / x, r_{y}=-2 x q .
\end{gathered}
$$

A solution of system (5.1) is

$$
p=1, q=0, r=x^{2} .
$$

To obtain the Cartesian coordinates $u(x, y)$ and $v(x, y)$, one has to solve ([10])

$$
\begin{gathered}
u_{x}^{2}+v_{x}^{2}=1, \\
u_{x} u_{y}+v_{x} v_{y}=0, \\
u_{y}^{2}+v_{y}^{2}=x^{2},
\end{gathered}
$$

Thus

$$
\begin{aligned}
& u=x \cos y, \\
& v=x \sin y,
\end{aligned}
$$

are the transformations that linearize the system.

2. The system which has $P=0, Q=-3, R=0, S=0, T=0, U=9 y^{2}, V=18, W=-6 / y^{2}$, is linearizable since it holds conditions (3.13)-(3.15) with $a, b, c, d, e$ and $f$ given in the Appendix. In the same manner as in example 1, one can obtain

$$
p=1+x^{2}-2 x / y+1 / y^{2}
$$




$$
\begin{gathered}
q=\left(1+x^{2}\right) / y^{2}-x / y^{3}, \\
r=\left(1+x^{2}\right) / y^{4} .
\end{gathered}
$$

For the coordinates transformations one can solve system (5.2) with the help of $p, q, r$ to obtain

$$
\begin{gathered}
u=x-1 / y, \\
v=x^{2} / 2-x / y .
\end{gathered}
$$

3. The system of two third order equations with constant coefficients $P=-2, Q=0, R=$ $-6, S=0, T=0, U=-6, V=0, W=-2$ is also linearizable as the determinant of (4.1) is zero.

The coordinate transformation for the above system which gives the linearization is

$$
\begin{aligned}
& u=c_{1} e^{-y-x}+c_{2} e^{y-x}, \\
& v=c_{1} e^{-y-x}-c_{2} e^{y-x} .
\end{aligned}
$$

Note that there are four arbitrary constants which will appear in the solution for each of the above examples and therefore we have conditional linearizability subject to a system of two second-order equations.

\section{Concluding Remarks}

In this paper we have provided conditional linearizability criteria for a class of third-order systems of ODEs. To the best of our knowledge the linearization of systems of third-order ODEs has not been studied before in the literature. The system of two third-order ODEs that is cubically semi-linear in the first derivative was obtained by differentiating a system of two quadratically semi-linear ODEs and then we used the original system to replace the second derivative. The resultant system was then not a total derivative.

The criteria developed are discussed in two parts. In the first part we constructed the linearizability conditions when at least one of the coefficients is a non-constant function, so that the system

obtained for the linearizability conditions be invertible. For the constant coefficients case we 
obtain a system which is independent of the derivatives. The non-trivial solution of the resulting system requires that the determinant of the coefficients be zero. There were various sub-cases. The linearizability conditions for each of the sub-cases of constant coefficients were constructed. This procedure will hopefully give rise to further studies in the construction of linearizability criteria for systems of higher-order equations.

\section{Acknowledgements}

AQ is most grateful to DECMA and the School of Computational and Applied Mathematics, University of the Witwatersrand and for some useful comments by Profs. P. Leach, S. Maleshko and R. Popovych.

\section{Appendix}

By using Mathematica one can find $a, b, c, d, e$ and $f$ from system (3.15) in terms of $P, Q, R, S, T, U, V$ and $W$ as

$$
\begin{gathered}
a=\frac{1}{\Delta}\left[3 \left(-9 S^{2} T U^{2}+R S U^{3}+27 S^{2} T^{2} V-Q S U^{2} V-3 Q S T V^{2}+3 P S U V^{2}\right.\right. \\
-27 R S T^{2} W+18 Q S T U W-Q R U^{2} W+3 Q R T V W-27 P S T V W+Q^{2} U V W \\
\left.\left.-3 P R U V W-9 Q^{2} T W^{2}+27 P R T W^{2}\right)\left(3 P_{y}-Q_{x}\right)\right)+2\left(9 R S T U^{2}-R^{2} U^{3}\right. \\
-27 R S T^{2} V+2 Q R U^{2} V-9 P S U^{2} V+27 P S T V^{2}-Q^{2} U V^{2}+27 R^{2} T^{2} W \\
\left.-18 Q R T U W+9 P R U^{2} W+9 Q^{2} T V W-27 P R T V W\right)\left(Q_{y}-R_{x}\right) \\
+3(Q T-P U)\left(-3 S U^{2}+9 S T V+R U V-Q V^{2}-9 R T W+3 Q U W\right)\left(R_{y}-3 S_{x}\right) \\
-3\left(\left(Q R S U^{2}-9 P S^{2} U^{2}-3 Q R S T V+27 P S^{2} T V-Q{ }^{2} S U V+3 P R S V\right.\right. \\
+3 Q R^{2} T W-27 P R S T W-Q^{2} R U W-3 P R^{2} U W+18 P Q S U W+Q^{3} V W \\
\left.-27 P^{2} S V W-9 P Q^{2} W W^{2}+27 P^{2} R W^{2}\right)\left(3 T_{y}-U_{x}\right)+2\left(Q R^{2} U^{2}\right. \\
-9 P R S U^{2}-9 Q^{2} S T V+27 P R S T V-2 Q^{2} R U V+18 P Q S U V+Q^{3} V^{2} \\
\left.-27 P^{2} S V^{2}+9 Q^{2} R T W-27 P R^{2} T W-9 P Q^{2} V W+27 P^{2} R V W\right)\left(U_{y}-V_{x}\right) \\
\left.-3(Q T-P U)\left(R^{2} U-3 Q S U-Q R V+9 P S V+3 Q^{2} W-9 P R W\right)\left(V_{y}-3 W_{x}\right)\right] \\
+R^{2} U^{2} W-6 Q S U^{2} W-3 R^{2} T V W+9 P S U V W-Q^{2} V^{2} W+3 P R V^{2} W+3 Q^{2} U W^{2} \\
b=\frac{1}{\Delta}\left[-3\left(3 S^{2} U^{3}-9 S^{2} T U V-2 R S U^{2} V+3 R S T V^{2}+2 Q S U V^{2}-3 P S V^{3}+9 R S T U W\right.\right.
\end{gathered}
$$




$$
\begin{aligned}
& \left.-9 P R U W^{2}\right)\left(3 P_{y}-Q_{x}\right)-2(R U-Q V)\left(-3 S U^{2}+9 S T V+R U V-Q V^{2}-9 R T W\right. \\
& +3 Q U W)\left(Q_{y}-R_{x}\right)-(R U-Q V)\left(R U^{2}-3 R T V-Q U V+3 P V^{2}+9 Q T W\right. \\
& -9 P U W)\left(R_{y}-3 S_{x}\right)-3\left(R^{2} S U^{2}-3 Q S^{2} U^{2}-3 R^{2} S T V+9 Q S^{2} T V-Q^{2} S V^{2}\right. \\
& +3 P R S V^{2}+3 R^{3} T W-9 Q R S T W-2 Q R^{2} U W+6 Q^{2} S U W+2 Q^{2} R V W-3 P R^{2} V W \\
& \left.-9 P Q S V W-3 Q^{3} W^{2}+9 P Q R W^{2}\right)\left(3 T_{y}-U_{x}\right)+2(R U-Q V)\left(R^{2} U-3 Q S U-Q R V\right. \\
& \left.+9 P S V+3 Q^{2} W-9 P R W\right)\left(U_{y}-V_{x}\right)-(R U-Q V)\left(3 R^{2} T-9 Q S T-Q R U\right. \\
& \left.\left.+9 P S U+Q^{2} V-3 P R V\right)\left(V_{y}-3 W_{x}\right)\right], \\
& c=\frac{1}{\Delta}\left[-3(S V-R W)\left(3 S U^{2}-9 S T V-R U V+Q V^{2}+9 R T W-3 Q U W\right)\left(3 P_{y}-Q_{x}\right)\right. \\
& -6(-S V+R W)\left(R U^{2}-3 R T V-Q U V+3 P V^{2}+9 Q T W-9 P U W\right)\left(Q_{y}-R_{x}\right) \\
& -\left(R^{2} U^{2} V-9 Q S T V^{2}-2 Q R U V^{2}+9 P S U V^{2}+Q^{2} V^{3}-9 R^{2} T U W+27 Q S T U W\right. \\
& \left.-27 P S U^{2} W+18 Q R T V W-9 P Q V^{2} W-27 Q^{2} T W^{2}+27 P Q U W^{2}\right)\left(R_{y}-3 S_{x}\right) \\
& +3(-S V+R W)\left(R^{2} U-3 Q S U-Q R V+9 P S V+3 Q^{2} W-9 P R W\right)\left(3 T_{y}-U_{x}\right) \\
& -6\left(3 R^{2} T-9 Q S T-Q R U+9 P S U+Q^{2} V-3 P R V\right)(-S V+R W)\left(U_{y}-V_{x}\right) \\
& +\left(-9 R^{2} S T U+27 Q S^{2} T U+R^{3} U^{2}-27 P S^{2} U^{2}-2 Q R^{2} U V+18 P R S U V+Q^{2} R V^{2}\right. \\
& \left.\left.-9 P Q S V^{2}+9 Q R^{2} T W-27 Q^{2} S T W-9 P R^{2} U W+27 P Q S U W\right)\left(V_{y}-3 W_{x}\right)\right], \\
& d=\frac{1}{\Delta}\left[9 R S T U^{2}-R^{2} U^{3}-27 R S T^{2} V+2 Q R U^{2} V-9 P S U^{2} V+27 P S T V^{2}-Q^{2} U V^{2}\right. \\
& \left.+27 R^{2} T^{2} W-18 Q R T U W+9 P R U^{2} W+9 Q^{2} T V W-27 P R T V W\right)\left(3 P_{y}-Q_{x}\right) \\
& +6(Q T-P U)\left(-3 S U^{2}+9 S T V+R U V-Q V^{2}-9 R T W+3 Q U W\right)\left(Q_{y}-R_{x}\right) \\
& +3(Q T-P U)\left(R U^{2}-3 R T V-Q U V+3 P V^{2}+9 Q T W-9 P U W\right)\left(R_{y}-3 S_{x}\right)+Q R^{2} U^{2} \\
& -9 P R S U^{2}-9 Q^{2} S T V+27 P R S T V-2 Q^{2} R U V+18 P Q S U V+Q^{3} V^{2}-27 P^{2} S V^{2} \\
& \left.+9 Q^{2} R T W-27 P R^{2} T W-9 P Q^{2} V W+27 P^{2} R V W\right)\left(3 T_{y}-U_{x}\right)-6(Q T \\
& -P U)\left(R^{2} U-3 Q S U-Q R V+9 P S V+3 Q^{2} W-9 P R W\right)\left(U_{y}-V_{x}\right) \\
& \left.+3(Q T-P U)\left(3 R^{2} T-9 Q S T-Q R U+9 P S U+Q^{2} V-3 P R V\right)\left(V_{y}-3 W_{x}\right)\right],
\end{aligned}
$$




$$
\begin{gathered}
e=\frac{1}{\Delta}\left[-(R U-Q V)\left(-3 S U^{2}+9 S T V+R U V-Q V^{2}-9 R T W+3 Q U W\right)\left(3 P_{y}-Q_{x}\right)\right. \\
-2(R U-Q V)\left(R U^{2}-3 R T V-Q U V+3 P V^{2}+9 Q T W-9 P U W\right)\left(Q_{y}-R_{x}\right) \\
-3\left(-R^{2} T U^{2}+3 Q S T U^{2}-3 P S U^{3}+3 R^{2} T^{2} V-9 Q S T^{2} V+9 P S T U V\right. \\
+2 P R U^{2} V+Q^{2} T V^{2}-6 P R T V^{2}-2 P Q U V^{2}+3 P^{2} V^{3}-3 Q^{2} T U W \\
\left.+3 P Q U^{2} W+9 P Q T V W-9 P^{2} U V W\right)\left(R_{y}-3 S_{x}\right)+(R U-Q V)\left(R^{2} U-3 Q S U\right. \\
\left.-Q R V+9 P S V+3 Q^{2} W-9 P R W\right)\left(3 T_{y}-U_{x}\right)-2(R U-Q V)\left(3 R^{2} T-9 Q S T\right. \\
\left.-Q R U+9 P S U+Q^{2} V-3 P R V\right)\left(U_{y}-V_{x}\right)+3\left(3 R^{3} T^{2}-9 Q R S T^{2}-2 Q R^{2} T U\right. \\
+3 Q^{2} S T U+9 P R S T+P R^{2} U^{2}-3 P Q S U^{2}+2 Q^{2} R T V-6 P R^{2} T V-P Q^{2} V^{2} \\
\left.\left.+3 P^{2} R V^{2}-3 Q^{3} T W+9 P Q R T W+3 P Q^{2} U W-9 P^{2} R U W\right)\left(V_{y}-3 W_{x}\right)\right] \\
\\
+\frac{1}{\Delta}\left[3(S V-R W)\left(R U^{2}-3 R T V-Q U V+3 P V^{2}+9 Q T W-9 P U W\right)\left(3 P_{y}-Q_{x}\right)\right. \\
-2\left(R^{2} U^{2} V-9 Q S T V^{2}-2 Q R U V^{2}+9 P S U V^{2}+Q^{2} V^{3}-9 R^{2} T U W+27 Q S T U W\right. \\
\left.-27 P S U^{2} W+18 Q R T V W-9 P Q V^{2} W-27 Q^{2} T W^{2}+27 P Q U W^{2}\right)\left(Q_{y}-R_{x}\right) \\
-3\left(-R^{2} T U V+3 Q S T U V-3 P S U^{2} V+Q R T V^{2}+P R U V^{2}-P Q V^{3}+9 R^{2} T^{2} W\right. \\
-27 Q S T^{2} W-3 Q R T U W+27 P S T U W+3 P R U^{2} W-18 P R T V W+9 P^{2} V^{2} W \\
\left.+27 P Q T W^{2}-27 P^{2} U W^{2}\right)\left(R_{y}-3 S_{x}\right)-3\left(3 R^{2} T-9 Q S T-Q R U+9 P S U+Q^{2} V\right. \\
-3 P R V)(-S V+R W)\left(3 T_{y}-U_{x}\right)+2\left(-9 R^{2} S T U+27 Q S^{2} T U+R^{3} U^{2}-27 P S^{2} U^{2}\right. \\
-2 Q R^{2} U V+18 P R S U+Q^{2} R V^{2}-9 P Q S V^{2}+9 Q R^{2} T W-27 Q^{2} S T W-9 P R^{2} U W \\
+27 P Q S U W)\left(U_{y}-V_{x}\right)-3\left(-9 R^{2} S T^{2}+27 Q S^{2} T^{2}+R^{3} T U-27 P S^{2} T U-Q R^{2} T V\right. \\
-3 Q^{2} S T V+18 P R S T V-P R^{2} U V+3 P Q S U V+P Q R V^{2}-9 P^{2} S V^{2}+3 Q^{2} R T W \\
\left.\left.-27 P Q S T W-3 P Q R U W+27 P^{2} S U W\right)\left(V_{y}-3 W_{x}\right)\right], \\
+
\end{gathered}
$$

where

$$
\begin{gathered}
\Delta=\left(2 \left(-R^{3}\left(U^{3}-27 T^{2} W\right)-9 Q^{2}(2 S T+P W)\left(V^{2}-3 U W\right)+27 P S\left(S U^{3}-3 S T U V\right.\right.\right. \\
\left.-P V^{3}+3 P U V W\right)+Q^{3}\left(V^{3}-27 T W^{2}\right)+3 R^{2}\left(3 S T\left(U^{2}-3 T V\right)+6 P\left(U^{2}-3 T V\right) W\right. \\
+Q U(U V-9 T W)+27 Q S\left(S T\left(-U^{2}+3 T V\right)+P\left(U V^{2}-2 U^{2} W-3 T V W\right)\right) \\
+3 R\left(Q^{2} V(-U V+9 T W)-3 Q(S T-P W)(-U V+9 T W)+9 P\left(P W\left(V^{2}-3 U W\right)\right.\right. \\
\left.\left.\left.+S\left(-U^{2} V+2 T V^{2}+3 T U W\right)\right)\right)\right) .
\end{gathered}
$$




\section{References}

[1] Lie S. "Klassifikation und Integration von gewölicchen Differentialgleichungen zwischen $x, y$, die eine Gruppe von Transformationen gestaten", Arch. Math. VIII, IX 187 (1883).

[2] Lie S, Theorie der Transformationsgruppen, Math. Ann. (1880), 16441.

[3] Nayfeh A H, Perturbation Methods, Wiley-Interscience, New York, (1973).

[4] Chern S S, "Sur la geometrie d'une equation differentielle du troiseme orde", C. R. Acad. Sci. Paris, (1973) 1227.

[5] Chern S S, "The geometry of the differential equation $y^{\prime \prime \prime}=F\left(x, y, y^{\prime}, y^{\prime \prime}\right)$ ", Sci, Rep. Nat. Tsing Hua Univ., 4 (1940) 97-111.

[6] Grebot G, "The characterization of third order ordinary differential equations admitting a transitive fibre-preserving point symmetry group", J. Math. Anal. Applic., 206 (1997) 364-388.

[7] Neut S and Petitot M, "La geometrie de l'equation $y^{\prime \prime \prime}=f\left(x, y, y^{\prime}, y^{\prime \prime}\right)$ ", C. R. Acad. Sci. Paris Ser I, 335 (2002) 515-518.

[8] Ibragimov N H and Meleshko S V, "Linearization of third-order ordinary differential equations by point and contact transformations", J. Math. Anal. Applic. 308 (2005) 266-289.

[9] Meleshko S V, "On linearization of third-order ordinary differential equations", J. Phys. A.: Math. Gen. Math. 39 (2006), 15135-45.

[10] Mahomed F M and Qadir A, "Linearization criteria for a system of second-order quadratically semi-linear ordinary differential equations", Non-linear Dynamics, 48, 417-422 (2007).

[11] Feroze T, Mahomed F M and Qadir A, "The connection between isometries and symmetries of geodesic equations of the underlying spaces", Nonlinear Dynamics, 45 65-74 (2006).

[12] Aminova A V and Aminov N A -M, "Projective geometry of systems of differential equations: general conceptions", Tensor N.S, 62 (2000) 65.

[13] Mahomed F M and Qadir A, "Invariant linearization criteria for systems of cubically semilinear second-order ordinary differential equations", Journal of Non-linear Mathematical Physics, to appear.

[14] Mahomed F M and Qadir A, "Linearizability criteria for a lass of third order semi-linear ordinary differential equations", preprint. 